\title{
Hip Extensor Strength Influences Dynamic Postural Changes during Gait in Patients with Adult Spinal Deformity: A Cross-Sectional Study Using Three-Dimensional Motion Analysis
}

\author{
Keita Sato ${ }^{1}$, Ryoji Tominaga ${ }^{1}$, Tatsuya Endo ${ }^{1}$, Takuya Miura ${ }^{1}$, \\ Masumi Iwabuchi ${ }^{1}$, Toshikazu Ito ${ }^{2}$, Osamu Shirado ${ }^{1}$ \\ ${ }^{1}$ Departments of Rehabilitation/Orthopaedic and Spinal Surgery, Aizu Medical Center, Fukushima Medical University, Aizuwakamatsu, Japan \\ ${ }^{2}$ Hokkaido Chitose College of Rehabilitation, Chitose, Japan
}

Study Design: Single-hospital cross-sectional study.

Purpose: The aim of the present study was to investigate the physical functions influencing dynamic postural change in patients with adult spinal deformity (ASD).

Overview of Literature: Dynamic postural change leading to increased forward lean during gait is a problem in patients with ASD; however, the relationship between this change and trunk and hip extensor strength is unclear.

Methods: Thirty patients with ASD aged $\geq 50$ years who were admitted to our hospital between July 2016 and September 2019 were included in this study. X-ray parameters (i.e., sagittal vertical axis, pelvic tilt, and pelvic incidence minus lumbar lordosis) were evaluated from the full-length standing radiographs of the subjects. Trunk and hip extensor strength was evaluated using a hand-held dynamometer. Dynamic postural changes (i.e., sagittal trunk shift during standing, sagittal trunk shift during gait, and delta sagittal trunk shift) were assessed using a three-dimensional motion analysis system. The relationships between dynamic postural change and various $\mathrm{X}$-ray parameters, as well as trunk and hip extensor strength, were examined using multivariable analysis.

Results: Multivariable analysis showed that hip extensor strength is the factor most strongly associated with dynamic postural change among the $X$-ray parameters and physical functions assessed in this study $\left(\beta=-0.41, R^{2}=0.12\right)$.

Conclusions: We demonstrated the association between dynamic postural change and hip extensor strength in patients with ASD. Our results may be useful to healthcare providers treating patients with ASD. Interventions for dynamic postural change in patients with ASD should focus on hip extensor strength.

Keywords: Spine; Adult spinal deformity; Sagittal alignment; Walking; Muscle strength

Received Jun 17, 2021; Revised Aug 26, 2021; Accepted Sep 14, 2021

Corresponding author: Keita Sato

Departments of Rehabilitation and Orthopaedic and Spinal Surgery, Aizu Medical Center, Fukushima Medical University, 21-2, Tanisawa-aza-maeda, Kawahigashi, Aizuwakamatsu, Fukushima, 969-3492, Japan

Tel: +81-242-75-2100, Fax: +81-242-75-2150, E-mail: keita-s@fmu.ac.jp 


\section{Introduction}

Adult spinal deformity (ASD), a musculoskeletal disorder, occurs in approximately $20 \%-40 \%$ of the Japanese elderly population and causes various medical problems [1-4]. Patients with ASD present with a number of physical functional impairments and increased risk of falls due to spinal malalignment [5-7]. ASD has also been reported to result in a decrease in quality of life (QOL) [8]. Evaluation of spinal alignment using standing radiographs has been correlated with QOL, and accurate assessment of the sagittal and coronal radiographic parameters of the whole spine is essential to determine treatment indications for patients with ASD $[9,10]$.

Some researchers have suggested that the standing radiographs used to assess spinal alignment in patients with ASD may not reflect the actual posture of the patient in real-life situations because standing requires effortful postural changes and compensatory mechanisms $[11,12]$. Thus, studies on spinal alignment assessment by gait analysis are increasingly being conducted using a threedimensional motion analysis system (3D-MAS); indeed, this technology has shown that the gait posture of patients with ASD demonstrates increased anteversion of the trunk and pelvis compared with the standing posture [1317]. Patients with ASD have also been reported to demonstrate increased anteversion when they walk even if they no longer manifest a poor standing posture. The results of these studies highlight the necessity of assessing patients with ASD from a dynamic perspective [18]. Dynamic postural changes associated with gait are a kinematic feature of patients with ASD and a factor that could exacerbate spinal alignment. However, to the best of our knowledge, no conclusive reports examining the causes of dynamic postural change as well as the relationships of the latter with various physical functions, such as muscle strength, have yet been published.

The aim of the present study was to investigate the physical functions influencing dynamic postural change in patients with ASD. We hypothesized that the trunk and hip extensors, which are essential to maintain posture, are associated with dynamic postural changes in patients with ASD. Knowledge of the physical functions involved in dynamic postural change in patients with ASD could reveal therapeutic strategies for improving postural abnormalities in such patients.

\section{Materials and Methods}

\section{Study design}

This cross-sectional study was performed at a single hospital. All study participants provided written informed consent prior to data collection. The Strengthening the Reporting of Observational Studies in Epidemiology guidelines were strictly followed [19]. This study was approved by the Ethics Committee of Fukushima Medical University (IRB approval no., 29359).

\section{Patient population}

Patients with ASD who were admitted to admitted to Aizu Medical Center, Fukushima Medical University (Aizuwakamatsu, Japan) for operative or conservative treatment between July 2016 and September 2019 were enrolled to this study under the following inclusion criteria: aged $\geq 50$ years and at least one of the following measures of spinal deformity: (1) a sagittal vertical axis (SVA) of $40 \mathrm{~mm}$ or greater, (2) a pelvic tilt (PT) of $20^{\circ}$ or greater, or (3) a pelvic incidence minus lumbar lordosis value (PI-LL) of $10^{\circ}$ or greater [9]. Patients with neuromuscular diseases, deformities due to infections or tumors of the spine, and hip or knee pain due to osteoarthritis of the lower limbs were excluded from this study. We also excluded patients with lumbar spinal canal stenosis who had nerve root symptoms or leg pain associated with walking, such as neurogenic intermittent claudication, as well as those with respiratory or cardiovascular diseases, which could affect gait performance.

\section{Measurement of X-ray parameters}

$\mathrm{X}$-ray parameter measurements were conducted by two spinal surgeons using full-length standing radiographs, with the patient in the fist-on-clavicle position. The following spinopelvic parameters were measured: (1) SVA, i.e., the distance between the $\mathrm{C} 7$ plumb line and the posterior superior corner on the top margin of S1; (2) lumbar lordosis (LL), i.e., the angle between the superior endplate of L1 and the superior endplate of S1; (3) PT, i.e., the angle between the line perpendicular to the center of the femoral head and the line connecting the center of the femoral head and the midpoint of the sacral endplate; (4) pelvic incidence (PI), i.e., the angle between the line 


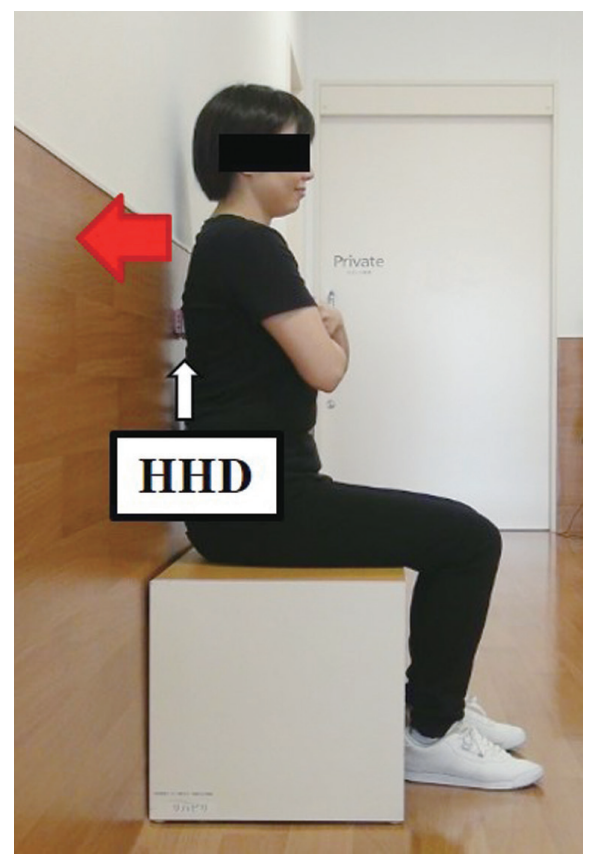

Fig. 1. Evaluation of trunk extensor strength for hand-held dynamometer (HHD). Measuring trunk extensor strength during maximum effort trunk extension in a seated position.

perpendicular to the sacral plate at its midpoint and the line connecting this point to the femoral head axis; and (5) PI-LL.

\section{Measurement of physical functions}

Trunk and hip extensor strength was assessed by measuring muscle strength with a hand-held dynamometer (HHD, Mobie MT-100; SAKAI Medical, Tokyo, Japan). Measurements were collected during isometric trunk and hip extension with maximum effort. Trunk extensor strength was measured in a sitting position with the HHD placed between the subject's back and the wall [20] (Fig. 1). Here, the patients were verbally instructed to push against the wall with their back with as much strength as possible without putting any effort into their legs. Hip extensor strength was measured in a sitting position on the treatment bed with the HHD placed between the distal thighs of the subjects (Fig. 2). Zero correction was performed by pressing the offset switch after the HHD was placed on the subjects' thighs to minimize the effect of the weight of the device on the measurement obtained [21]. Here, the patients were verbally instructed to push the treatment bed downward with their thighs with as much strength as possible.

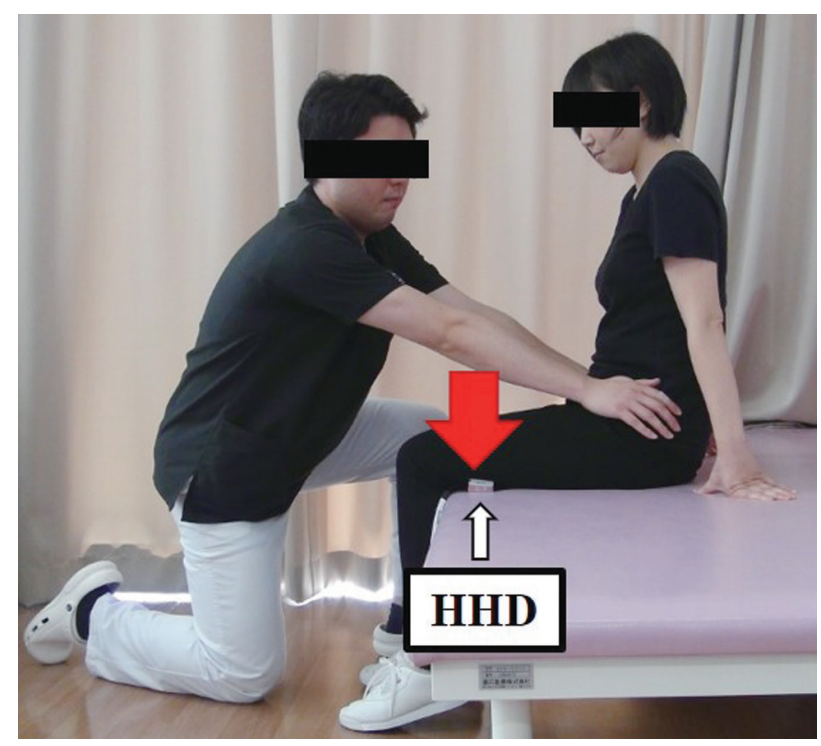

Fig. 2. Evaluation of hip extensor strength for hand-held dynamometer (HHD). Measuring hip extensor strength during maximum effort hip extension in a seated position.

\section{Evaluation of dynamic postural change using 3D-MAS}

Measurements of standing and gait postures were performed using a 3D-MAS instrument (VICON Motion Capture Systems, Oxford, UK) operated at a sampling rate of $200 \mathrm{~Hz}$. The walkway was approximately $8 \mathrm{~m}$ long, and eight infrared cameras were used. Two force plates (AMTI, Watertown, MA, USA) were installed at the midpoint of the walkway at $1,000 \mathrm{~Hz}$. Infrared markers were installed on $\mathrm{C} 7$ (i.e., on the spinous process of the seventh cervical vertebra) and S1 (i.e., on the spinous process of the first sacral vertebra). Sagittal trunk shift (STS) was calculated from the coordinate data obtained according to the method of Shiba et al. [13]. STS refers to the distance between the C7 and S1 markers in the sagittal plane and was measured in millimeters. The measurement obtained is a relative value that indicates the degree of displacement of the $\mathrm{C} 7$ marker relative to the $\mathrm{S} 1$ marker; it is expressed as a positive value $(+)$ when the $\mathrm{C} 7$ marker is located in front of the $\mathrm{S} 1$ marker and as a negative value (-) when it is located behind the S1 marker. STS was measured in the standing (standing STS) and gait (gait STS) postures. Standing data were measured over a period of 5 seconds from the start of standing. Here, the patients' arms were lowered along their body, and their gaze was directed forward. Gait data were measured at the time of gait initiation. The data collected over one gait cycle by 3D-MAS were normalized to $100 \%$. Filtering (fourth-order Butterworth low-pass filter; $6 \mathrm{~Hz}$ ) 


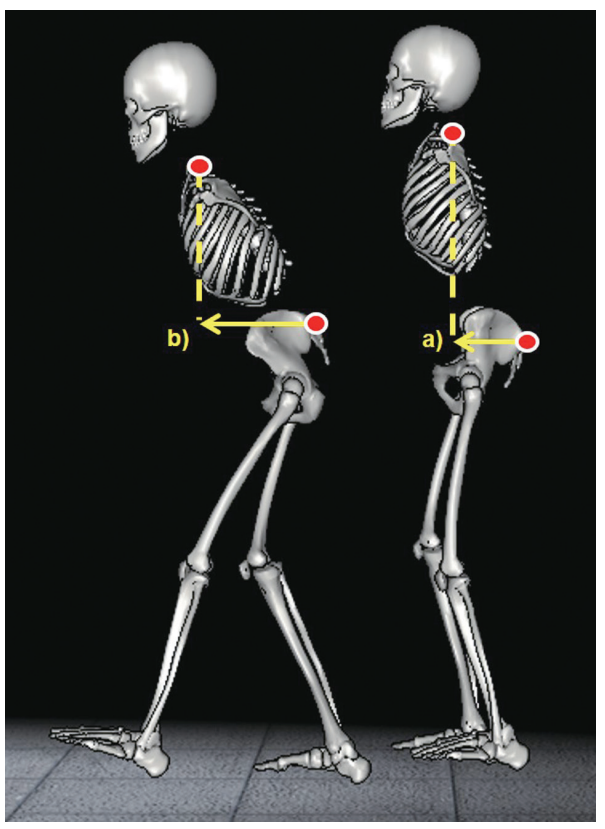

Fig. 3. Standing and gait posture assessment using three-dimensional motion analysis system (3D-MAS; VICON Motion Capture Systems, Oxford, UK). ${ }^{\text {a) }}$ Sagittal trunk shift during standing (standing sagittal trunk shift [STS]). ${ }^{\text {b) Sagittal }}$ trunk shift during gait (gait STS). The greater the anterior trunk tilt, the greater the STS value. Postural change is shown and defined as $\triangle$ STS

was applied to the marker coordinate data and the analog data obtained from the floor reaction meter for smoothing. Initial contact was defined as the first point at which the ground reaction force in the vertical direction exceeded $10 \mathrm{~N}$. Toe off was defined as the first point at which the ground reaction force in the vertical direction dropped to $10 \mathrm{~N}$ or less. STS was measured under the following conditions: (1) gait at self-selected speed, (2) adequate breaks and practice, and (3) average of the data measured over 5 seconds of standing and an average of three gait analyses. Dynamic postural change $(\Delta \mathrm{STS})$, which was defined as the STS during gait minus the STS during standing, was then calculated. Dynamic postural change reflects a change in posture associated with the onset of gait initiation from a standing position (Fig. 3).

\section{Statistical analysis}

The data were analyzed using R Commander ver. 2.8.1 (freeware; The R Foundation for Statistical Computing, Vienna, Austria). All values are expressed as mean and standard deviation. The relationships between the $\Delta$ STS and each X-ray parameter and trunk and hip extensor strength of the subjects were determined by univariable
Table 1. Background data for the subjects

\begin{tabular}{lr} 
Characteristic & Mean \pm SD \\
\hline Age $(\mathrm{yr})$ & $72.8 \pm 8.2$ \\
\hline Height $(\mathrm{cm})$ & $147.9 \pm 5.9$ \\
\hline Weight $(\mathrm{kg})$ & $50.8 \pm 8.1$ \\
\hline Body mass index $\left(\mathrm{kg} / \mathrm{m}^{2}\right)$ & $23.2 \pm 3.5$ \\
\hline Sagittal vertical axis $(\mathrm{mm})$ & $154.9 \pm 79.9$ \\
\hline Pelvic tilt $\left(^{\circ}\right)$ & $39.0 \pm 10.0$ \\
\hline PI-LL $\left(^{\circ}\right)$ & $45.9 \pm 22.2$ \\
\hline Standing STS $(\mathrm{mm})$ & $88.3 \pm 73.5$ \\
\hline Gait STS (mm) & $156.4 \pm 74.9$ \\
\hline STS $(\mathrm{mm})$ & $78.4 \pm 65.1$ \\
\hline
\end{tabular}

SD, standard deviation; PI-LL, pelvic incidence minus lumbar lordosis; standing STS, sagittal trunk shift during standing; gait STS, sagittal trunk shift during gait; $\triangle S T S$, delta sagittal trunk shift (gait STS minus standing STS).

analysis using Spearman's rank correlation coefficients. Multivariable analysis of items that were found to be significantly correlated with $\Delta$ STS was then conducted to investigate their impact on $\triangle$ STS with adjustment for confounding factors (e.g., age and body mass index [BMI]). A $p<0.05$ was considered statistically significant.

\section{Results}

Thirty patients with ASD were included in this study. All patients were female. The patients' basic characteristics were as follows: age, $72.8 \pm 8.2$ years; height, $147.9 \pm 5.9 \mathrm{~cm}$; weight, $50.8 \pm 8.1 \mathrm{~kg}$; and BMI, $23.2 \pm 3.5 \mathrm{~kg} / \mathrm{m}^{2}$. The sagittal plane parameters acquired from the full-length standing lateral radiographs were as follows: SVA, $154.9 \pm 79.9 \mathrm{~mm}$; PT, $39.0^{\circ} \pm 10.0^{\circ}$; and PI-LL $45.9^{\circ} \pm 22.2^{\circ}$. The average kinematic parameters were as follows: standing STS, 88.3 \pm 73.5 $\mathrm{mm}$; gait STS, $156.4 \pm 74.9 \mathrm{~mm}$; and $\Delta \mathrm{STS}, 78.4 \pm 65.1 \mathrm{~mm}$ (Table 1).

Univariable analysis revealed a significant correlation between hip extensor strength and $\Delta$ STS ( $r=-0.40$, $p=0.03$ ). No significant correlation was found between $\triangle$ STS and any X-ray parameter (SVA: $r=-0.01, p=0.96$; PT: $r=0.17, p=0.38$; PI-LL: $r=0.05, p=0.78)$. Trunk extensor strength and X-ray parameters (SVA: $r=-0.25, p=0.19$; PT: $r=-0.06, p=0.75$; PI-LL: $r=-0.50, p=0.01$ ), hip extensor strength and X-ray parameters (SVA: $r=-0.19, p=0.31$; PT: $r=-0.25, p=0.19$; PI-LL: $r=-0.31, p=0.09$ ), and trunk extensor strength and PI-LL showed significant relationships (Table 2). Multivariable analysis indicated that hip 
Table 2. Relationship of X-ray parameters and physical functions to $\Delta S T S$

\begin{tabular}{lccccc} 
Variable & $\Delta S T S$ & SVA & PT & PI-LL & $\begin{array}{c}\text { Trunk extensor } \\
\text { strength }\end{array}$ \\
SVA & -0.01 & & & & \\
PT & 0.17 & $0.42^{*}$ & & & \\
PI-LL & 0.05 & $0.76^{*}$ & $0.41^{*}$ & & \\
Trunk extensor strength & -0.25 & -0.25 & -0.06 & $-0.50^{*}$ & \\
\hline Hip extensor strength & $-0.40^{*}$ & -0.19 & -0.25 & -0.31 & $0.49^{* *}$
\end{tabular}

Data are Spearman's rank correlation coefficients. Significance level was set at $5 \%$.

$\triangle$ STS, delta sagittal trunk shift; SVA, sagittal vertical axis; PT, pelvic tilt; PI-LL, pelvic incidence minus lumbar lordosis.

${ }^{*} p<0.05 .{ }^{* *} p<0.01$.

Table 3. Factors affecting dynamic postural changes in patients with adult spinal deformity

\begin{tabular}{lcccc} 
& $R^{2}$ & $B$ & $\beta$ & $p$-value \\
Hip extensor strength & 0.12 & -5.75 & -0.41 & $<0.05$ \\
\hline
\end{tabular}

Results in multivariable analysis. Significance level was set at $5 \%$.

$R^{2}$, coefficient of determination (adjusted); $B$, regression coefficients; $\beta$, standardized regression coefficients.

extensor strength is a major factor influencing increases in $\Delta$ STS $\left(\beta=-0.41, R^{2}=0.12\right)$ (Table 3).

\section{Discussion}

In the current study, we found that hip extensor strength is strongly associated with $\triangle \mathrm{STS}$. To the best of our knowledge, this study is the first to report the relationship between $\Delta$ STS and physical function. Increased $\Delta$ STS induces dynamic postural changes in patients with ASD, which strongly indicates that the function of the hip extensors during postural maintenance is important for such patients.

Spinal alignment in patients with ASD was assessed by standing radiographs. However, this technique has been reported to feature some limitations because standing necessitates effortful postural changes by the patient [11]. Therefore, 3D-MAS, which can well capture real-life situations, was used to evaluate dynamic spinal alignment [1118]. An earlier report on dynamic spinal alignment in patients with ASD showed that trunk and pelvis anteversion increases during gait compared with standing and that trunk and hip extensor strength may be involved in the postural change [13]. Arima et al. [18] reported that patients with ASD may not have a poor standing posture but show strong anteversion during gait. The results of these authors suggest the need to evaluate spinal alignment in patients with ASD from a dynamic perspective. Lee et al. [22] reported that large preoperative dynamic postural changes could negatively impact postoperative clinical outcomes. These reports emphasize the importance of dynamic postural changes in patients with ASD; however, factors related to dynamic postural changes, such as physical function, have not been examined thoroughly [13-18,22]. The current study builds on the findings of previous studies and extends the evidence for dynamic postural change in patients with ASD.

The current study revealed an association between dynamic postural change and hip extensor strength in patients with ASD. The trunk and pelvis are known to contribute more to anteversion during gait than during standing [13-17]. The hip extensors, such as the gluteus maximus and hamstrings, originate from the pelvic girdle and attach to the posterior aspect of the femur, which undergoes pelvic retroversion and prevents excessive pelvic anteversion [23]. The hip extensors of patients with ASD, along with the trunk extensors, are closely related to spinal sagittal alignment, and the degree of sagittal imbalance is well known to relate to the degree of muscle degeneration [24]. Therefore, hip extensor muscle weakness in patients with ASD may lead to excessive pelvic anteversion, which could be a factor for dynamic postural change. Another noteworthy finding in this study is the absence of a relationship between $\Delta$ STS and X-ray parameters. Radiological evaluation of patients with ASD is conventionally performed using full-length standing radiographs. However, this evaluation often masks the true posture of the patient because standing requires effortful postural changes and various compensatory mechanisms $[11,25]$. Pelvic retroversion in the standing posture of patients with ASD, for example, is one such compensatory mechanism [25]. Kim et al. [17] reported that posterior PT in patients with ASD disappears during walking, which suggests that this compensatory mechanism during standing is disrupted during gait. In other words, dynamic postural changes determined as $\Delta$ STS may represent events that differ from the features detected by fulllength standing radiographs. The results of this study may be useful for the treatment of patients with ASD in clinical practice, especially when the main complaint is gait disturbance, such as increased trunk anteversion during gait. Rehabilitation programs for patients with ASD often focus on trunk extensors [26-28]. The results of this study 
suggest the need to assess hip extensor strength if patients with ASD wish to improve their gait disturbance. Muscle strengthening exercises for hip extensors may also be considered as a method of rehabilitation for patients with ASD. In general, the greater the degree of preoperative dynamic postural change in patients with ASD, the poorer the postoperative clinical outcome [22]. If such patients scheduled for surgery present extensive dynamic postural change, preoperative strengthening exercises of the hip extensors may be useful. The results of this study suggest that hip extensor strengthening exercises could be added to the preoperative rehabilitation program of patients with ASD scheduled for surgery. However, the effectiveness of such exercises in patients with ASD and the development of specific rehabilitation programs must be investigated in future research.

This study features several strengths. First, 3D-MAS was employed to assess standing and gait posture in patients with ASD. Postural and motion analyses via 3D-MAS have been reported to yield highly reliable results. 3D-MAS allows observation of changes in posture in real time, which is difficult to achieve using radiological parameters alone. Second, the present study is the first to analyze the relationship between dynamic postural changes and physical functions in patients with ASD. Although previous studies have analyzed dynamic spinal alignment in patients with ASD, the present research actually quantifies and analyzes trunk and hip muscle strength, which are important considerations in spinal alignment.

The present study also includes a number of limitations. First, the presence of type 2 errors due to the small sample size cannot be excluded. Although a large sample size is desirable in any study, type 2 errors are generally considered a problem when the results of the analysis do not fall below the significance level. The results of this study showed significant associations, and the effect of type 2 errors on the analyses may be minimal. Second, continuous postural changes in gait were not assessed in this work. Dynamic postural changes in this study were analyzed at the initiation of gait, but evaluating postural changes in continuous gait may be necessary to understand the gait disorder of patients with ASD completely. Third, muscle activity during gait was not assessed. The muscle strength assessed in the current study differs from the actual muscle activity manifested during gait; specifically, in this work, only the maximum muscle strength the subject could exert was evaluated. Measurements of muscle activ- ity using surface electromyography may provide a quantitative indication of the real-time activity of muscles during movement. Surface electromyography could provide detailed insights into the relationship between real-time muscle activity and dynamic postural changes, which, in turn, could lead to a better understanding of dynamic postural changes in patients with ASD.

\section{Conclusions}

In conclusion, we demonstrated the association between dynamic postural changes and hip extensor strength in patients with ASD. This finding will be useful to physicians, physical therapists, and other healthcare professionals treating patients with ASD. Interventions for dynamic postural change in such patients should focus on hip extensor strength.

\section{Conflict of Interest}

No potential conflict of interest relevant to this article was reported.

\section{Acknowledgments}

All the participants in this study, the staff who assisted in writing and proofreading the paper, and the staff who supported this study.

\section{References}

1. Diebo BG, Shah NV, Boachie-Adjei O, et al. Adult spinal deformity. Lancet 2019;394:160-72.

2. Schwab F, Lafage V, Patel A, Farcy JP. Sagittal plane considerations and the pelvis in the adult patient. Spine (Phila Pa 1976) 2009;34:1828-33.

3. Kado DM, Browner WS, Palermo L, Nevitt MC, Genant HK, Cummings SR. Vertebral fractures and mortality in older women: a prospective study: study of Osteoporotic Fractures Research Group. Arch Intern Med 1999;159:1215-20.

4. Kobayashi T, Atsuta Y, Matsuno T, Takeda N. A longitudinal study of congruent sagittal spinal alignment in an adult cohort. Spine (Phila Pa 1976) 2004;29:671-6.

5. Schwab FJ, Blondel B, Bess S, et al. Radiographical spinopelvic parameters and disability in the setting 
of adult spinal deformity: a prospective multicenter analysis. Spine (Phila Pa 1976) 2013;38:E803-12.

6. Engsberg JR, Bridwell KH, Reitenbach AK, et al. Preoperative gait comparisons between adults undergoing long spinal deformity fusion surgery (thoracic to L4, L5, or sacrum) and controls. Spine (Phila Pa 1976) 2001;26:2020-8.

7. Kado DM, Huang MH, Nguyen CB, Barrett-Connor E, Greendale GA. Hyperkyphotic posture and risk of injurious falls in older persons: the Rancho Bernardo Study. J Gerontol A Biol Sci Med Sci 2007;62:652-7.

8. Glassman SD, Bridwell K, Dimar JR, Horton W, Berven S, Schwab F. The impact of positive sagittal balance in adult spinal deformity. Spine (Phila Pa 1976) 2005;30:2024-9.

9. Schwab F, Ungar B, Blondel B, et al. Scoliosis Research Society-Schwab adult spinal deformity classification: a validation study. Spine (Phila Pa 1976) 2012;37:1077-82.

10. Yilgor C, Sogunmez N, Boissiere L, et al. Global Alignment and Proportion (GAP) score: development and validation of a new method of analyzing spinopelvic alignment to predict mechanical complications after adult spinal deformity surgery. J Bone Joint Surg Am 2017;99:1661-72.

11. Marks MC, Stanford CF, Mahar AT, Newton PO. Standing lateral radiographic positioning does not represent customary standing balance. Spine (Phila Pa 1976) 2003;28:1176-82.

12. Bae J, Theologis AA, Jang JS, Lee SH, Deviren V. Impact of fatigue on maintenance of upright posture: dynamic assessment of sagittal spinal deformity parameters after walking 10 minutes. Spine (Phila $\mathrm{Pa}$ 1976) 2017;42:733-9.

13. Shiba Y, Taneichi H, Inami S, Moridaira H, Takeuchi D, Nohara Y. Dynamic global sagittal alignment evaluated by three-dimensional gait analysis in patients with degenerative lumbar kyphoscoliosis. Eur Spine J 2016;25:2572-9.

14. Miura K, Kadone H, Koda M, et al. Thoracic kyphosis and pelvic anteversion in patients with adult spinal deformity increase while walking: analyses of dynamic alignment change using a three-dimensional gait motion analysis system. Eur Spine J 2020;29:840-8.

15. Sasaki K, Hongo M, Miyakoshi N, et al. Evaluation of sagittal spine-pelvis-lower limb alignment in elderly women with pelvic retroversion while standing and walking using a three-dimensional musculoskeletal model. Asian Spine J 2017;11:562-9.

16. Mar DE, Kisinde S, Lieberman IH, Haddas R. Representative dynamic ranges of spinal alignment during gait in patients with mild and severe adult spinal deformities. Spine J 2021;21:518-27.

17. Kim HJ, Shen F, Kang KT, et al. Failure of pelvic compensation in patients with severe positive sagittal imbalance: comparison between static radiographs and gait analysis of spinopelvic parameters in adult spinal deformity and lumbar stenosis. Spine (Phila Pa 1976) 2019;44:E759-65.

18. Arima H, Yamato Y, Hasegawa T, et al. Discrepancy between standing posture and sagittal balance during walking in adult spinal deformity patients. Spine (Phila Pa 1976) 2017;42:E25-30.

19. von Elm E, Altman DG, Egger M, et al. The Strengthening the Reporting of Observational Studies in Epidemiology (STROBE) statement: guidelines for reporting observational studies. Int J Surg 2014;12:1495-9.

20. Takenaka H, Sugiura H, Kamiya M, et al. Predictors of walking ability after surgery for lumbar spinal canal stenosis: a prospective study. Spine J 2019;19:1824-31.

21. Seko T, Mori M, Ohnishi H, et al. Reliability and validity of seated hip extensor strength measurement by handheld dynamometer in older adults. J Geriatr Phys Ther 2019;42:E39-44.

22. Lee CS, Lee CK, Kim YT, Hong YM, Yoo JH. Dynamic sagittal imbalance of the spine in degenerative flat back: significance of pelvic tilt in surgical treatment. Spine (Phila Pa 1976) 2001;26:2029-35.

23. Pohtilla JF. Kinesiology of hip extension at selected angles of pelvifemoral extension. Arch Phys Med Rehabil 1969;50:241-50.

24. Bao H, Moal B, Vira S, et al. Spino-femoral muscles affect sagittal alignment and compensatory recruitment: a new look into soft tissues in adult spinal deformity. Eur Spine J 2020;29:2998-3005.

25. Lamartina C, Berjano P. Classification of sagittal imbalance based on spinal alignment and compensatory mechanisms. Eur Spine J 2014;23:1177-89.

26. Bansal S, Katzman WB, Giangregorio LM. Exercise for improving age-related hyperkyphotic posture: a systematic review. Arch Phys Med Rehabil 2014;95:129-40.

27. Itoi E, Sinaki M. Effect of back-strengthening exer- 
cise on posture in healthy women 49 to 65 years of age. Mayo Clin Proc 1994;69:1054-9.

28. Katzman WB, Vittinghoff E, Lin F, et al. Targeted spine strengthening exercise and posture training program to reduce hyperkyphosis in older adults: results from the study of hyperkyphosis, exercise, and function (SHEAF) randomized controlled trial. Osteoporos Int 2017;28:2831-41. 